\title{
Chemical fingerprinting and quantitative analysis of a Panax notoginseng preparation using HPLC-UV and HPLC-MS
}

\author{
Hong Yao, Peiying Shi, Qing Shao, Xiaohui Fan
}

\begin{abstract}
Background: Xuesaitong (XST) injection, consisting of total saponins from Panax notoginseng, was widely used for the treatment of cardio- and cerebro-vascular diseases in China. This study develops a simple and global quality evaluation method for the quality control of XST.

Methods: High performance liquid chromatography-ultraviolet detection (HPLC-UV) was used to identify and quantify the chromatographic fingerprints of the XST injection. Characteristic common peaks were identified using HPLC with photo diode array detection/electrospray ionization tandem mass spectrometry (HPLC-PDA/ESI-MS ${ }^{n}$ ).

Results: Representative fingerprints from ten batches of samples showed 27 'common saponins' all of which were identified and quantified using ten reference saponins.

Conclusion: Chemical fingerprinting and quantitative analysis identified most of the common saponins for the quality control of $P$. notoginseng products such as the XST injection.
\end{abstract}

\section{Background}

Xuesaitong (XST) injection, consisting of total saponins from Panax notoginseng (Sanqi), was widely used for the treatment of cardiovascular and cerebrovascular diseases in China. As total saponins (including ginsenosides and notoginsenosides) in the XST injection are its active ingredients, quality control of total saponins in the XST injection is critical for its safety, efficacy and stability. Single or simultaneous determination of main components of the total saponin extracts from $P$. notoginseng using high performance liquid chromatography-ultraviolet detection (HPLC-UV) [1-5], high performance liquid chromatography-evaporative light scattering detection (HPLC-ELSD) [6], high performance liquid chromatography-mass spectroscopy (HPLC-MS) [7-13] have been reported but over half of the total saponins were not quantified in these studies due to the lack of saponin references or poor chromatographic resolution. A comprehensive and systematic quality control of saponin extracts is much needed.

\footnotetext{
* Correspondence: fanxh@zju.edu.cn
Pharmaceutical Informatics Institute, Zhejiang University, Hangzhou 310058,

* Correspondence: fanxh@zju.edu.cn China
}

(c) 2011 Yao et al; licensee BioMed Central Ltd. This is an Open Access article distributed under the terms of the Creative Commons Attribution License (http://creativecommons.org/licenses/by/2.0), which permits unrestricted use, distribution, and reproduction in any medium, provided the original work is properly cited.
Fingerprint analysis is currently developed for quality control in Chinese medicine [14-26] and has been accepted by the WHO for the assessment of herbal medi(SFDA) of China requires all herbal medicine-derived injections and related materials to use chromatographic fingerprints [28] in standardization.

This article reports a novel fingerprint analytical method for quality control of the XST injection, which may be applicable to other herbal products. Over the previous studies [1-13], the new method features the following advantages. (1) The representative fingerprints show good chromatographic separation for most of visible peaks in the chromatographic profiles at $203 \mathrm{~nm}$; (2) All main saponins (27 visible peaks in chromatographic profiles) are identifiable using high performance liquid chromatography-photo diode array detection/electrospray ionization tandem mass spectrometry (HPLC-PDA/ESI-MS ${ }^{\mathrm{n}}$ ) technique, ten saponin references or data from literature [8-14].

\section{Methods}

Materials and reagents

Acetonitrile and methanol (HPLC grade) were purchased from Merck (Darmstadt, Germany). Acetic acid cines [27]. The State Food and Drug Administration 
glacial (HPLC grade) was from Tedia (Fairfield, OH, USA). The water used was purified by Milli-Q system (Millipore, USA). Reference compounds, namely notoginsenoside $\mathrm{R}_{1}$, ginsenoside $\mathrm{Rg}_{1}, \mathrm{Rg}_{2}, \mathrm{Rh}_{1}, \mathrm{Rb}_{1}, \mathrm{Rb}_{2}, \mathrm{Rd}$, Re, $20(\mathrm{~S})-\mathrm{Rg}_{3}$ and $20(\mathrm{R})-\mathrm{Rg}_{3}$ were purchased from Jilin University (Shenyang, China). The structures of these compounds are shown in Figure 1. Mixed standard stock solution containing accurately weighed reference compounds was directly prepared in $80 \%$ aqueous methanol $(\mathrm{v} / \mathrm{v})$. Working standard solutions were prepared by diluting the stock solution with $80 \%$ aqueous methanol $(\mathrm{v} / \mathrm{v})$ to obtain a series of concentrations for the calibration curves.

\section{HPLC instrumentationadditional 1 and chromatographic conditions}

An Agilent 1100 HPLC system (Agilent Technologies, USA) consisted of a quaternary solvent delivery system, an on-line degasser, an auto-sampler, a column temperature controller and ultraviolet detector coupled with an analytical workstation and an Ultimate ${ }^{\mathrm{TM}} \mathrm{XB}-\mathrm{C}_{18}$ column, $5 \mu \mathrm{m}, 250 \mathrm{~mm} \times 4.6 \mathrm{~mm}$ i.d. (Welch Materials, USA) were used in the HPLC-UV experiments. Flow rate was $1.0 \mathrm{ml} / \mathrm{min}$ and sample injection volume was $10 \mu \mathrm{l}$. Detection wavelength was set at $203 \mathrm{~nm}$ and the column temperature was at $30^{\circ} \mathrm{C}$. Mobile phase contained deionized water-acetic acid (A; 100:0.01, v/v) and acetonitrile-acetic acid (B; 100:0.01, v/v). The gradient elution was as follows: $19-21.2 \% \mathrm{~B}$ at $0-30 \mathrm{~min} ; 21.2-$ $26 \% \mathrm{~B}$ at $30-35 \mathrm{~min} ; 26-28 \% \mathrm{~B}$ at $35-40 \mathrm{~min} ; 28-38 \% \mathrm{~B}$ at $40-50 \mathrm{~min} ; 38-55 \% \mathrm{~B}$ at $50-60 \mathrm{~min} ; 55 \% \mathrm{~B}$ at $60-65$ $\mathrm{min} ; 55-80 \% \mathrm{~B}$ at $65-70 \mathrm{~min} ; 80-95 \% \mathrm{~B}$ at $70-75 \mathrm{~min}$. Re-equilibrium was $10 \mathrm{~min}$; the total run time was 85 min.

\section{HPLC-MS $^{n}$ instrumentation and chromatographic conditions}

Analysis was performed on an Agilent 1100 series LC system equipped with a binary solvent delivery system, an auto-sampler, a column temperature controller, a photo diode array detector and a Finnigan LCQ Deca $\mathrm{XP}^{\text {plus }}$ ion trap mass spectrometer (Thermo Finnigan, USA) via an ESI interface. The chromatographic conditions were the same for HPLC-UV as described in the previous section. The operating parameters for MS in the negative mode were as follows: collision gas, ultrahigh-purity helium (He); nebulizing gas, high purity nitrogen $\left(\mathrm{N}_{2}\right)$; ion spray voltage, $-4.5 \mathrm{kV}$; sheath gas $\left(\mathrm{N}_{2}\right)$ at a flow rate of 60 arbitrary units; auxiliary gas $\left(\mathrm{N}_{2}\right)$ at a flow rate of 20 arbitrary units; capillary temperature, $350^{\circ} \mathrm{C}$; capillary voltage, $-15 \mathrm{~V}$; tube lens offset voltage, $-30 \mathrm{~V}$. Full scan data acquisition was performed from $\mathrm{m} / z 80$ to 1800 in MS scan mode. The MS ${ }^{\mathrm{n}}$ spectra were obtained with the collision energy for collision- induced dissociation adjusted to $30 \%-40 \%$ of maximum and the isolation width of precursor ions was 2.0Th.

\section{Sample preparation}

Ten samples of the XST injection (Batch No. 20090307, 20090510, 20090310, 20081018, 9042213, 20090312, 20090421, 20090512, 20090504, 20090203), manufactured by three Chinese pharmaceutical companies, were obtained either from pharmacies or factories. For HPLC-PDA-MS ${ }^{\mathrm{n}}$ analysis, a certain volume of the injection, according to its nominal content of total saponins, was transferred to a $50 \mathrm{ml}$ volumetric flask and was diluted with $80 \%$ aqueous methanol $(\mathrm{v} / \mathrm{v})$ to obtain total saponins at a concentration of about $1 \mathrm{mg} / \mathrm{ml}$. For HPLC-UV analysis, the injection was diluted with $80 \%$ aqueous methanol $(\mathrm{v} / \mathrm{v})$ to obtain total saponins at a concentration of about $0.5 \mathrm{mg} / \mathrm{ml}$. Prior to analysis, the sample solutions were filtered through a $0.45 \mu \mathrm{m}$ nylon membrane (Whatman, Britain). Spiked injection was produced by mixing sample solutions with the reference solutions at the ratio of 1:1.

\section{Data analysis}

Data analysis was carried out with Similarity Evaluation System for Chromatographic Fingerprint of Traditional Chinese Medicine (version 2004A, National Committee of Pharmacopoeia, China) recommended by the SFDA.

\section{Results and discussion}

\section{Optimization of HPLC separation}

We optimized the separation conditions including the column, mobile phase, detection wavelength, elution gradient and column temperature in this study. Four reversed-phase columns, Agilent Zorbax Eclipse SB- $\mathrm{C}_{18}$ columns $(250 \mathrm{~mm} \times 4.6 \mathrm{~mm}, 5 \mu \mathrm{m} ; 150 \mathrm{~mm} \times 4.6 \mathrm{~mm}$, $3.5 \mu \mathrm{m} ; 100 \mathrm{~mm} \times 2.1 \mathrm{~mm}, 1.8 \mu \mathrm{m})$ and Ultimate ${ }^{\mathrm{TM}}$ XB- $\mathrm{C}_{18}$ column $(250 \mathrm{~mm} \times 4.6 \mathrm{~mm}, 5 \mu \mathrm{m})$ were tested. The results showed that all four columns obtained good peak resolutions in $75 \mathrm{~min}, 75 \mathrm{~min}, 45 \mathrm{~min}$ and $75 \mathrm{~min}$ respectively; however, only two columns with the length of $250 \mathrm{~mm}$ (Zorbax Eclipse SB- $\mathrm{C}_{18}$ and Ultimate ${ }^{\mathrm{TM}} \mathrm{XB}$ $\mathrm{C}_{18}$ ) produced more peaks in chromatograms. Ultimate $^{\mathrm{TM}} \mathrm{XB}-\mathrm{C}_{18}$ column $(250 \mathrm{~mm} \times 4.6 \mathrm{~mm}, 5 \mu \mathrm{m})$ was selected in the fingerprint analysis due to its lower cost than Zorbax Eclipse SB- $\mathrm{C}_{18}$ column.

The effects of mobile phase composition on chromatographic separation were also studied. The cetonitrile/ water system produced more sharp peaks than the methanol/water system; the addition of $0.01 \%$ acetic acid in the acetonitrile/water system further improved the peak shape. Moreover, as the retention time of some components such as ginsenoside $20(\mathrm{~S})-\mathrm{Rg}_{3}$ and $20(\mathrm{R})$ $\mathrm{Rg}_{3}$ was long, gradient elution was used in HPLC analysis. Satisfactory separation was achieved in $75 \mathrm{~min}$. 


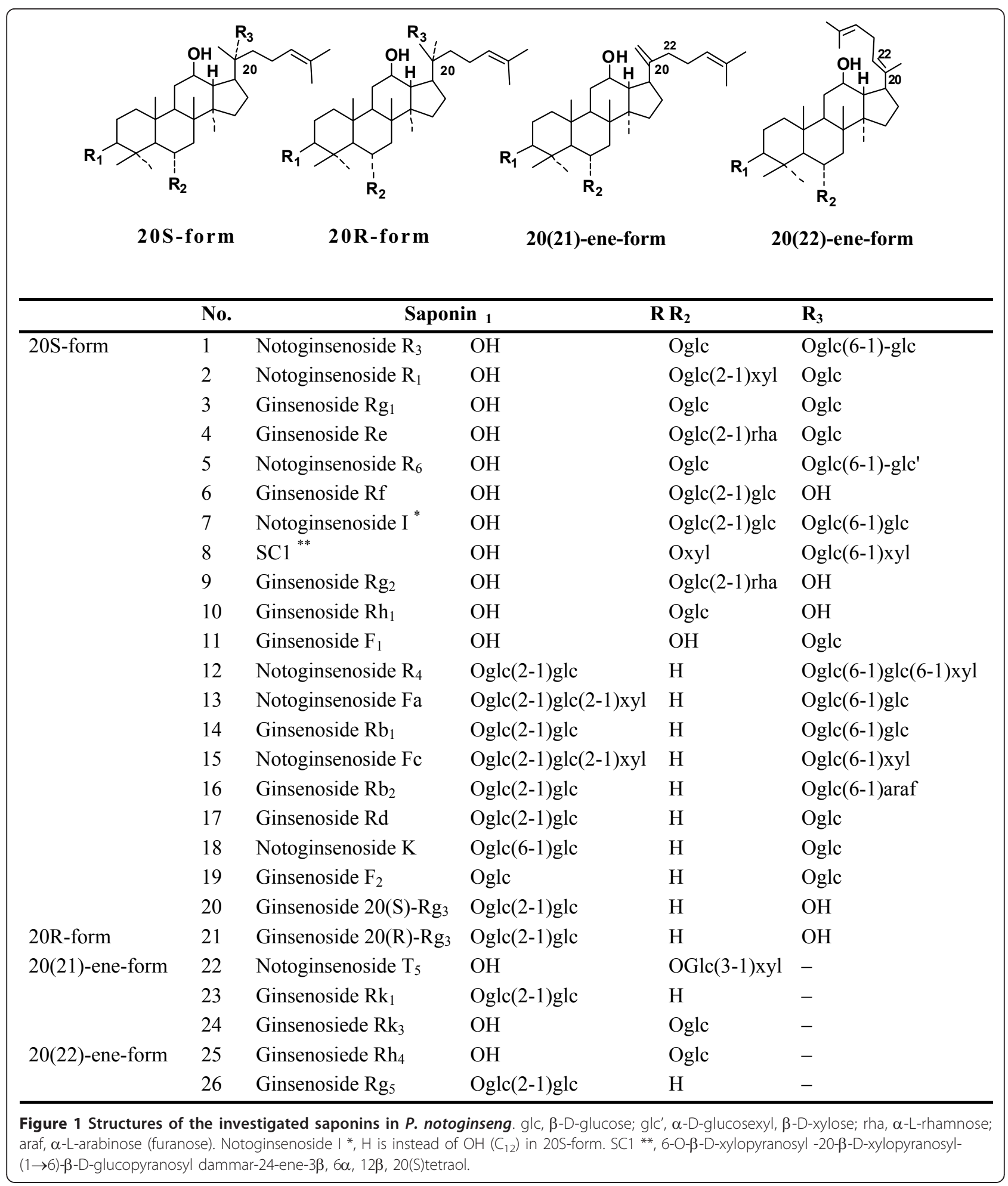

There was no strong absorption for most of saponins in the region of ultraviolet and visible spectra due to their structural characteristics, eg lack of conjugation groups in the molecular structures. As the end adsorption wavelength $203 \mathrm{~nm}$ is suitable for the assay of ginsenosides and notoginsenosides [1-5], it was selected as the detection wavelength in the experiment. Furthermore, the effects of column temperature on chromatographic separation were also examined. Four column temperatures, namely $20,25,30$ and $35^{\circ} \mathrm{C}$ were tested. 
We found that at $30^{\circ} \mathrm{C}$ most peaks in chromatography had good resolutions; therefore, $30^{\circ} \mathrm{C}$ was chosen as the column temperature for the fingerprint analysis.

\section{HPLC-UV fingerprinting of the XST injection}

To standardize the fingerprints, we analyzed ten samples using the optimized HPLC-UV method. Peaks found in all ten samples with good resolution were assigned as 'characteristic peaks' and there were 27 characteristic peaks in the fingerprint chromatograms (Figure 2A). The software of Similarity Evaluation System for Chromatographic Fingerprint of Traditional Chinese Medicine was used to evaluate these chromatograms. To exclude the effects of the solvent and baseline fluctuation, we selected the chromatographic data of these ten samples and treated them within the time frame of $28 \mathrm{~min}$ to $75 \mathrm{~min}$. The similarities of chromatograms for the ten samples to the reference fingerprints were established using the means of all chromatograms (Additional file 1). The results showed that the ten samples possessed similarities to the reference fingerprints (Additional file 2). While the HPLC-UV fingerprints from different batches and companies varied, the 27 characteristic peaks were common in all samples. Therefore, the detection of these common peaks in HPLC fingerprints is useful in assessing the quality of the XST injection.

\section{Identification of characteristic peaks}

HPLC-PDA/ESI-MS ${ }^{\mathrm{n}}$ was used for the components analysis and all 27 characteristic peaks were identified. In the ESI-MS experiment, the molecular weight of each peak was also obtained. By comparing with the ESI-MS ${ }^{\mathrm{n}}$ data and HPLC retention time of standard sanponins (Figure 2B and Additional file 3), we identified 10 peaks as notogisenoside
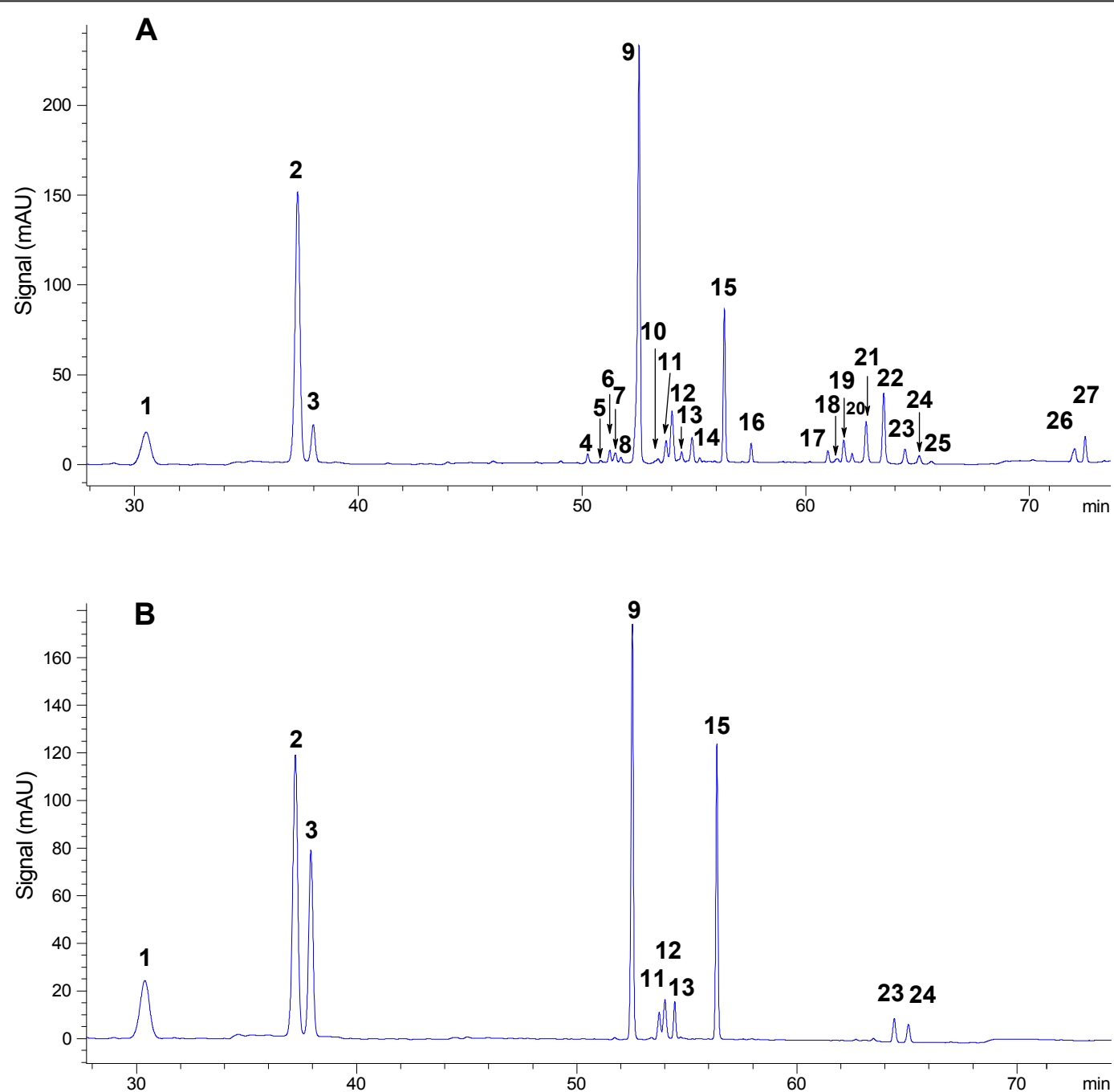

Figure 2 Chromatograms of (A) the representative fingerprint, (B) mixture standard compounds including (1) notoginsenoside $\mathrm{R}_{1}$, (2) ginsenoside $R g_{1}$, (3) ginsenoside $R e$, (9) ginsenoside $R b_{1}$, (11) ginsenoside $R g_{2}$, (12) ginsenoside $R h_{1}$, (13) ginsenoside $R b_{2}$, (15), ginsenoside $\mathrm{Rd},(23)$ ginsenoside $20(\mathrm{~S})-\mathrm{Rg}_{3}$ and (24) ginsenoside $20(\mathrm{R})-\mathrm{Rg}_{3}$. 
$R_{1}$, ginsenoside $R g_{1}, R e, R_{1}, R g_{2}, R h_{1}, R b_{2}$, Rd and 20(S)$R_{3}, 20(R)-R_{3}$. A total of 17 peaks were identified tentatively with the aid of the ESI-MS ${ }^{\mathrm{n}}$ data and HPLC retention time of some saponins from previous reports [1-13]. All the identification results are shown in Table 1 . In addition, The UV spectra of all peaks in the XST injection were obtained from the PDA chromatogram (Additional file 3). The results showed that among all the peaks in the chromatogram of the XST injection no strong UV absorption within the wavelength range from $210 \mathrm{~nm}$ to $400 \mathrm{~nm}$ was obtained, suggesting that the XST injection consisted of saponins with few other natural components possessing strong UV absorption, such as flavonoids, lignins, anthraquinones and alkaloids.

Determination of the main saponins in the XST injection As shown in Figure 2A, 27 saponins were well separated, of which 25 were potentially identified (Table 1 ). The ratio of total saponin peak area to all peaks (except for solvent peaks and baseline fluctuation in $0-28 \mathrm{~min}$ ) in the chromatogram of each sample was beyond $95 \%$. Thus, a method for quantification of the 27 saponins should provide a global and systematical evaluation for the quality control of the XST injection. However, it was difficult to obtain the reference compounds for all 27 saponins; we were only able to obtain ten, including notoginsenoside $R_{1}$, ginsenoside $R g_{1}, R e, R_{1}, R g_{2}, R h_{1}, R b_{2}, R d, 20(S)-R g_{3}$ and $20(\mathrm{R})-\mathrm{Rg}_{3}$. Some reports [1-3] found that the slopes of regression equations for most of the determined saponins, such as notoginsenoside $\mathrm{R}_{2}, \mathrm{R}_{4}, \mathrm{Fa}$, ginsenoside $\mathrm{Rg}_{1}, \mathrm{Re}$, $R f, b_{1}, R g_{2}, R h_{1}$ and $R d$ were approximately negatively correlated to their molecular weights by HPLC-UV at 203 $\mathrm{nm}$ (Additional file 4) and that the regression equations of some saponins with similar molecular weights were also close to each other under the same chromatographic condition (Additional file 5, 6, 7, 8 and 9).

Ten saponins, namely $R_{1}$, ginsenoside $\mathrm{Rg}_{1}, \mathrm{Re}, \mathrm{Rb}_{1}, \mathrm{Rg}_{2}$, $R h_{1}, R b_{2}, R d, 20(S)-R_{3}$ and $20(R)-R_{3}$ were quantitatively determined and the rest 17 saponins without standard references were semi-quantified using substitutive

Table 1 The identification results of saponins in the XST injection by LC/MS

\begin{tabular}{|c|c|c|c|c|}
\hline $\begin{array}{l}\text { Peak } \\
\text { No. }\end{array}$ & Identification & $\begin{array}{l}\text { Retention time } \\
(\mathrm{min})\end{array}$ & $\mathrm{MS}[\mathrm{M}-\mathrm{H}]^{-}$ & MS data $(m / z)$ \\
\hline 1 & Notoginsenoside $\mathrm{R}_{1}$ & 34.89 & 932 & $799[\mathrm{M}-\mathrm{H}-\mathrm{Xyl}] ; 637[\mathrm{M}-\mathrm{H}-\mathrm{Xyl}-\mathrm{GlC}] ; 475 \mathrm{Agl}$ \\
\hline 2 & Ginsenoside $\mathrm{Rg}_{1}$ & 39.32 & 800 & $637[\mathrm{M}-\mathrm{H}-\mathrm{GlC}] ; 619\left[\mathrm{M}-\mathrm{H}-\mathrm{H}_{2} \mathrm{O}-\mathrm{GlC}\right] ; 475 \mathrm{Agl}$ \\
\hline 3 & Ginsenoside Re & 39.72 & 945 & 783 [M-H-Glc]; 637 [M-H-Glc-Rha]; $475 \mathrm{Agl}$ \\
\hline 4 & Notoginsenoside $\mathrm{R}_{4}$ & 51.24 & 1240 & $1107[\mathrm{M}-\mathrm{H}-\mathrm{Xyl}]^{-} ; 1077$ [M-H-Glc]]; $945[\mathrm{M}-\mathrm{H}-\mathrm{Xyl}-\mathrm{Glc} ; 783$ [M-H-Xyl-2Glc] \\
\hline 5 & Ginsenoside Rf & 51.89 & 800 & $637[\mathrm{M}-\mathrm{H}-\mathrm{GlC}]]^{-} ; 475 \mathrm{Agl}$ \\
\hline 6 & Notoginsenoside $\mathrm{Fa}$ & 52.17 & 1240 & $\left.1107[\mathrm{M}-\mathrm{H}-\mathrm{Xyl}]^{-} ; 1077[\mathrm{M}-\mathrm{H}-\mathrm{Glc}]\right]^{-} ; 945[\mathrm{M}-\mathrm{H}-\mathrm{Xyl}-\mathrm{Glc} ; 783$ [M-H-Xyl-2Glc] \\
\hline 7 & Notoginsenoside I & 52.39 & 1092 & $929[\mathrm{M}-\mathrm{H}-\mathrm{GlC}]^{-} ; 767[\mathrm{M}-\mathrm{H}-2 \mathrm{GlC}]^{3} ; 605[\mathrm{M}-\mathrm{H}-3 \mathrm{GlC}]^{-}$ \\
\hline 8 & $\mathrm{SC} 1$ & 52.56 & 901 & 769 [M-H-Xyl]; 637 [M-H-2Xyl]; 475 Agl \\
\hline 9 & Ginsenoside Rb1 & 53.48 & 1107 & $945[\mathrm{M}-\mathrm{H}-\mathrm{GlC}] ; 783[\mathrm{M}-\mathrm{H}-2 \mathrm{GlC}] ; 621[\mathrm{M}-\mathrm{H}-3 \mathrm{Glc}] ; 459 \mathrm{Agl}$ \\
\hline 10 & Notoginsenoside Fc & 54.32 & 1209 & $\begin{array}{l}1077[\mathrm{M}-\mathrm{H}-\mathrm{Xyl}] ; 945[\mathrm{M}-\mathrm{H}-2 \mathrm{Xyl}] ; 783[\mathrm{M}-\mathrm{H}-2 \mathrm{Xyl}-\mathrm{GlC}] ; 621[\mathrm{M}-\mathrm{H}-2 \mathrm{Xyl}-2 \mathrm{Glc}] \\
; 459 \mathrm{Agl}\end{array}$ \\
\hline 11 & Ginsenoside $\mathrm{Rg}_{2}$ & 54.75 & 783 & $637[\mathrm{M}-\mathrm{H}-\mathrm{Rha}]^{-} ; 621[\mathrm{M}-\mathrm{H}-\mathrm{GlC}]^{-} ; 475 \mathrm{Agl}$ \\
\hline 12 & Ginsenoside $\mathrm{Rh}_{1}$ & 55.04 & 637 & $475[\mathrm{M}-\mathrm{H}-\mathrm{GlC}]^{-}$ \\
\hline 13 & Ginsenoside $\mathrm{Rb}_{2}$ & 55.30 & 1077 & $\begin{array}{l}\text { 945[M-H-Arap]; } 915[\mathrm{M}-\mathrm{H}-\mathrm{Glc}] ; \text {; } 783[\mathrm{M}-\mathrm{HArap}-\mathrm{Glc}] ; \text {; } 621[\mathrm{M}-\mathrm{H}-\text { Arap-2Glc]; } \\
459 \mathrm{Agl}\end{array}$ \\
\hline 14 & Ginsenoside $F_{1}$ & 55.84 & 637 & $475[\mathrm{M}-\mathrm{H}-\mathrm{GlC}]^{-}$ \\
\hline 15 & Ginsenoside Rd & 57.16 & 945 & 783 [M-H-Glc]; 621[M-H-2Glc]; 459Agl \\
\hline 16 & Notoginsenoside K & 58.32 & 945 & 783 [M-H-Glc]; $621[\mathrm{M}-\mathrm{H}-2 \mathrm{Glc}]^{-} ; 459 \mathrm{Agl}$ \\
\hline 17 & $\begin{array}{l}\text { Notoginsenoside } T_{5} / \\
\text { Unkown }\end{array}$ & 61.70 & 752 & $619[\mathrm{M}-\mathrm{H}-\mathrm{Xyl}]^{-} ; 457 \mathrm{Agl}$ \\
\hline 18 & Unkown & 62.09 & 765 & $603[\mathrm{M}-\mathrm{H}-\mathrm{GlC}]^{-}$ \\
\hline 19 & $\begin{array}{l}\text { Notoginsenoside } T_{5} / \\
\text { Unkown }\end{array}$ & 62.42 & 752 & $619[\mathrm{M}-\mathrm{H}-\mathrm{Xyl}] ; 457 \mathrm{Agl}$ \\
\hline 20 & Unkown & 62.81 & 765 & $603[\mathrm{M}-\mathrm{H}-\mathrm{GlC}]^{-}$ \\
\hline 21 & Ginsenoside $\mathrm{Rk}_{3}$ & 63.42 & 619 & $551\left[\mathrm{M}-\mathrm{H}-\mathrm{C}_{5} \mathrm{H}_{10}\right]^{-}$ \\
\hline 22 & Ginsenoside $\mathrm{Rh}_{4}$ & 64.18 & 619 & $551\left[\mathrm{M}-\mathrm{H}-\mathrm{C}_{5} \mathrm{H}_{10}\right]^{-}$ \\
\hline 23 & 20(S)-ginsenoside $\mathrm{Rg}_{3}$ & 65.14 & 783 & $621[\mathrm{M}-\mathrm{H}-\mathrm{GlC}]^{-} ; 459 \mathrm{Agl}$ \\
\hline 24 & 20(R)-ginsenoside $\mathrm{Rg}_{3}$ & 65.86 & 783 & $621[\mathrm{M}-\mathrm{H}-\mathrm{GlC}]^{-} ; 459 \mathrm{Agl}$ \\
\hline 25 & Ginsenoside $F_{2}$ & 66.05 & 783 & 621 [M-H-GlC]; 459 Agl \\
\hline 26 & Ginsenoside $\mathrm{Rk}_{1}$ & 72.47 & 765 & $603[\mathrm{M}-\mathrm{H}-\mathrm{GlC}]^{-}$ \\
\hline 27 & Ginsenoside $\mathrm{Rg}_{5}$ & 72.89 & 765 & $603[\mathrm{M}-\mathrm{H}-\mathrm{GlC}]^{-}$ \\
\hline
\end{tabular}


standard substances. The calibration curves for the quantification of each saponin were selected and listed in Table 2. The developed analytical method was successfully applied to analysis of ten batches of the XST injection. All of the 27 characteristic peaks were determined simultaneously and the results are in Table 3 . In the XST injection, the content of ginsenoside $\mathrm{Rb}_{1}$ was the most (26.17\%-29.60\%), followed by ginsenoside $\operatorname{Rg}_{1}$ (20.50\%$25.43 \%)$, Rd (6.82\%-8.10\%), notoginsenoside $\mathrm{R}_{1}$ (5.29\%$6.89 \%)$ and ginsenoside $\operatorname{Re}(2.91 \%-4.92 \%)$. The total content of the five saponins made up $61.69 \%-71.39 \%$ of the total saponins in the XST injection (total saponins nominal: $50 \mathrm{mg} / \mathrm{ml}$ ). The ten saponins with available standard substances were quantitatively determined and made up $68.46 \%-75.85 \%$ of the total saponins nominal. Thus, combined with the semi-quantification data, 81.81\%-95.71\% components in the XST injection could be examined.

\section{Conclusion}

The fingerprint profiles of ten batches of samples showed 27 characteristic peaks. Ten of these 27 saponins in the XST injections were quantitatively determined with their standard references; the rest 17 saponins were semiquantified with the substitutive standard references.

\section{Additional material}

\section{Additional file 1: The chromatogram of similarity analysis of the} fingerprints of 10 samples.

Additional file 2: The similarities of chromatograms of 10 samples ( $n=3)$.

Additional file 3: PDA Chromatograms. standard compounds (A) and a XST injection (C), and total ion current chromatograms of standard compounds (B) and a XST injection (D). 1-27 were the characteristic peaks, listed in Table 2

Additional file 4: Plots of slopes of calibration curves vs. molecular weights (MW) of saponins. From literatures (A) [Journal of

Pharmaceutical and Biomedical Analysis 41 (2006) 274-279], (B) [Journal of Pharmaceutical and Biomedical Analysis 48 (2008) 1361-1367], (C) [Journal of Pharmaceutical and Biomedical Analysis 38 (2005) 45-51], (D) [Journal of Chromatography A 1011 (2003) 77-87], (E) [Journal of Shenyang Pharmaceutical University Vol. 20, No.1 (2003) 27-31], and (F) [Chinese Pharmaceutical Journal Vol. 38, No.9 (2003) 698-699]

Additional file 5: The method validation for simultaneous determination of the twenty-seven saponins in XST injection. The quantitative and semi-quantitative methods were validated and the semi-quantitative principle were discussed in detail.

Table 2 Calibration curves, detection limits and quantification limits of the saponins by HPLC-UV

\begin{tabular}{|c|c|c|c|c|c|c|}
\hline Peak No. & Saponins & M.W. & Calibration curve $^{a}$ & Linear range $(\mu \mathrm{g} / \mathrm{ml})$ & $R^{2}$ & LOD $(\mu \mathrm{g} / \mathrm{ml})$ \\
\hline 21 & Ginsenoside $\mathrm{Rk}_{3}$ & 619 & $y=6.7519 x-7.6085$ & & & \\
\hline 22 & Ginsenoside $\mathrm{Rh}_{4}$ & 619 & $y=6.7519 x-7.6085$ & & & \\
\hline 12 & Ginsenoside $\mathrm{Rh}_{1}$ & 637 & $y=6.7519 x-7.6085$ & $4.28-68.5$ & 0.9993 & 2.14 \\
\hline 14 & Ginsenoside $F_{1}$ & 637 & $y=6.7519 x-7.6085$ & & & \\
\hline 17 & Notoginsenoside $T_{5}$ /Unkown & 752 & $y=5.4845 x-4.8387$ & & & \\
\hline 19 & Notoginsenoside $T_{5} /$ Unkown & 752 & $y=5.4845 x-4.8387$ & & & \\
\hline 18 & Unkown & 765 & $y=5.4845 x-4.8387$ & & & \\
\hline 20 & Unkown & 765 & $y=5.4845 x-4.8387$ & & & \\
\hline 26 & Ginsenoside $\mathrm{Rk}_{1}$ & 765 & $y=5.4845 x-4.8387$ & & & \\
\hline 27 & Ginsenoside $\mathrm{Rg}_{5}$ & 765 & $y=5.4845 x-4.8387$ & & & \\
\hline 11 & Ginsenoside $\mathrm{Rg}_{2}$ & 783 & $y=5.6715 x-5.6679$ & $3.34-53.5$ & 0.9993 & 1.67 \\
\hline 23 & $20(S)-\mathrm{Rg}_{3}$ & 783 & $y=5.4845 x-4.8387$ & $2.95-47.3$ & 0.9990 & 1.48 \\
\hline 24 & $20(R)-R_{3}$ & 783 & $y=5.0923 x-2.8995$ & $2.63-42.0$ & 0.9994 & 1.75 \\
\hline 25 & Ginsenoside $F_{2}$ & 783 & $y=5.4845 x-4.8387$ & & & \\
\hline 2 & Ginsenoside $\mathrm{Rg}_{1}$ & 800 & $y=5.1367 x-76.471$ & 16.64-1065 & 0.9990 & 10.29 \\
\hline 5 & Ginsenoside Rf & 800 & $y=5.1367 x-76.471$ & & & \\
\hline 8 & SC1 & 901 & $y=4.3254 x-5.0843$ & & & \\
\hline 1 & Notoginsenoside $R_{1}$ & 932 & $y=4.3254 x-5.0843$ & $10.26-492.5$ & 0.9997 & 7.42 \\
\hline 3 & Ginsenoside Re & 945 & $y=4.4123 x-29.465$ & $43.28-692.5$ & 0.9993 & 4.73 \\
\hline 15 & Ginsenoside Rd & 945 & $y=4.1199 x-5.5681$ & $16.64-532.5$ & 0.9993 & 4.43 \\
\hline 16 & Notoginsenoside K & 945 & $y=4.1199 x-5.5681$ & & & \\
\hline 13 & Ginsenoside $\mathrm{Rb}_{2}$ & 1077 & $y=3.8757 x+2.4182$ & $4.84-77.5$ & 0.9995 & 1.95 \\
\hline 7 & Notoginsenoside I & 1092 & $y=3.8757 x+2.4182$ & & & \\
\hline 9 & Ginsenoside $\mathrm{Rb}_{1}$ & 1107 & $y=3.5815 x-29.548$ & $15.98-1022.5$ & 0.9992 & 7.91 \\
\hline 10 & Notoginsenoside Fc & 1209 & $y=3.5815 x-29.548$ & & & \\
\hline 4 & Notoginsenoside $R_{4}$ & 1240 & $y=3.5815 x-29.548$ & & & \\
\hline 6 & Notoginsenoside Fa & 1240 & $y=3.5815 x-29.548$ & & & \\
\hline
\end{tabular}

${ }^{a} \mathrm{y}$ : peak area of analyte; $\mathrm{x}$ : concentration of analyte $(\mu \mathrm{g} / \mathrm{ml})$. 
Table 3 Contents (\%) of the 27 saponins in the XST injection (total saponins nominal: $50 \mathrm{mg} / \mathrm{ml})^{\mathrm{a}}$

\begin{tabular}{|c|c|c|c|c|c|c|c|c|c|c|c|}
\hline Peak No. & Saponins & S1 & S2 & S3 & S4 & S5 & S6 & S7 & S8 & S9 & S10 \\
\hline 1 & Notoginsenoside $\mathrm{R}_{1}(\%)$ & 6.64 & 5.29 & 6.89 & 6.47 & 6.27 & 5.86 & 5.33 & 6.41 & 6.07 & 6.35 \\
\hline 2 & Ginsenoside $\operatorname{Rg}_{1}(\%)$ & 25.43 & 20.50 & 24.53 & 23.99 & 23.76 & 20.29 & 21.15 & 22.23 & 22.31 & 23.33 \\
\hline 3 & Ginsenoside Re (\%) & 3.43 & 2.91 & 4.92 & 3.61 & 3.55 & 3.56 & 3.35 & 3.04 & 3.03 & 3.69 \\
\hline 4 & Notoginsenoside $\mathrm{R}_{4}(\%)$ & 1.52 & 1.19 & 1.24 & 1.33 & 1.28 & 1.33 & 1.31 & 1.11 & 1.15 & 1.38 \\
\hline 5 & Ginsenoside Rf (\%) & 1.24 & 0.95 & 0.98 & 1.15 & 1.15 & 0.97 & 1.03 & 1.03 & 1.03 & 1.00 \\
\hline 6 & Notoginsenoside Fa (\%) & 1.45 & 1.21 & 1.90 & 1.35 & 1.44 & 1.43 & 1.35 & 1.29 & 1.29 & 1.34 \\
\hline 7 & Notoginsenoside I (\%) & 0.89 & 0.62 & 0.17 & 0.80 & 0.80 & 0.76 & 0.81 & 0.73 & 0.66 & 0.83 \\
\hline 8 & SC1 (\%) & 0.65 & 0.51 & 2.28 & 0.56 & 0.62 & 0.46 & 0.54 & 0.52 & 0.49 & 0.54 \\
\hline 9 & Ginsenoside $\mathrm{Rb}_{1}(\%)$ & 28.39 & 26.17 & 26.34 & 28.30 & 28.78 & 29.58 & 29.60 & 28.00 & 28.14 & 27.78 \\
\hline 10 & Notoginsenoside Fc (\%) & 1.30 & 0.94 & 0.99 & 1.13 & 1.12 & 1.06 & 0.98 & 1.05 & 1.05 & 1.15 \\
\hline 11 & Ginsenoside $\mathrm{Rg}_{2}(\%)$ & 1.02 & 1.31 & 1.08 & 1.18 & 0.98 & 0.78 & 1.44 & 1.38 & 1.38 & 1.17 \\
\hline 12 & Ginsenoside $\mathrm{Rh}_{1}(\%)$ & 1.77 & 3.06 & 2.25 & 2.22 & 1.65 & 1.06 & 2.90 & 3.19 & 3.22 & 2.17 \\
\hline 13 & Ginsenoside $\mathrm{Rb}_{2}(\%)$ & 1.09 & 0.69 & 2.18 & 1.07 & 1.06 & 1.00 & 0.90 & 0.81 & 1.11 & 1.04 \\
\hline 14 & Ginsenoside $F_{1}(\%)$ & 0.76 & 1.77 & 0.29 & 1.14 & 0.85 & 0.50 & 1.59 & 1.90 & 1.88 & 1.24 \\
\hline 15 & Ginsenoside Rd (\%) & 7.50 & 6.82 & 7.25 & 7.22 & 7.24 & 7.27 & 8.10 & 7.41 & 7.48 & 7.18 \\
\hline 16 & Notoginsenoside K (\%) & 1.01 & 0.72 & 1.05 & 1.18 & 1.24 & 1.33 & 1.36 & 0.96 & 1.04 & 1.43 \\
\hline 17 & Notoginsenoside $T_{5} /$ Unkown (\%) & 0.39 & 0.69 & 0.58 & 0.69 & 0.47 & 0.39 & 0.79 & 0.87 & 0.86 & 0.83 \\
\hline 18 & Unkown (\%) & 0.30 & 0.37 & 1.11 & 0.45 & 0.36 & 0.23 & 0.56 & 0.50 & 0.50 & 0.46 \\
\hline 19 & Notoginsenoside $T_{5} /$ Unkown (\%) & 0.72 & 1.31 & 0.41 & 1.19 & 0.82 & 0.63 & 1.51 & 1.51 & 1.54 & 1.20 \\
\hline 20 & Unkown (\%) & 0.39 & 0.55 & 0.31 & 0.55 & 0.37 & 0.39 & 0.70 & 0.66 & 0.67 & 0.55 \\
\hline 21 & Ginsenoside $\mathrm{Rk}_{3}(\%)$ & 0.90 & 2.30 & 1.59 & 1.78 & 1.10 & 0.80 & 2.35 & 2.52 & 2.57 & 1.77 \\
\hline 22 & Ginsenoside $\mathrm{Rh}_{4}(\%)$ & 1.27 & 3.66 & 2.47 & 2.69 & 1.49 & 0.91 & 3.70 & 3.87 & 3.88 & 2.65 \\
\hline 23 & $20 \mathrm{~S}-\mathrm{Rg}_{3}(\%)$ & 0.37 & 1.01 & 0.75 & 0.81 & 0.44 & 0.43 & 1.21 & 1.09 & 1.14 & 0.83 \\
\hline 24 & $20 R-\operatorname{Rg}_{3}(\%)$ & 0.21 & 0.70 & 0.52 & 0.51 & 0.25 & 0.22 & 0.78 & 0.76 & 0.82 & 0.56 \\
\hline 25 & Ginsenoside $F_{2}(\%)$ & 0.36 & 0.38 & 0.23 & 0.28 & 0.14 & 0.10 & 0.78 & 0.42 & 0.43 & 0.25 \\
\hline 26 & Ginsenoside $\mathrm{Rk}_{1}(\%)$ & 0.41 & 1.13 & 1.22 & 0.81 & 0.66 & 0.47 & 1.62 & 1.02 & 1.28 & 0.80 \\
\hline \multirow[t]{2}{*}{27} & Ginsenoside $\mathrm{Rg}_{5}(\%)$ & 0.32 & 1.30 & 1.17 & 1.05 & 0.65 & 0.46 & 1.95 & 1.31 & 1.50 & 1.03 \\
\hline & Total (\%) ${ }^{b}$ & 89.41 & 86.78 & 93.54 & 92.47 & 87.90 & 81.81 & 95.71 & 94.27 & 95.02 & 91.50 \\
\hline
\end{tabular}

a Mean values of samples $(n=3)$.

b Total content of the 27 saponins in samples.

Additional file 6: Precisions and repeatability. The results of precision and repeatability for simultaneous determination of the twenty-seven saponins

Additional file 7: Recovery. The results of recovery for simultaneous determination of the twenty-seven saponins

Additional file 8: Plots of slopes of calibration curves vs molecular weights (MW) with different chromatography columns. (A)

Ultimate ${ }^{\mathrm{TM}^{\mathrm{TM}}} \mathrm{XB}-\mathrm{C} 18(250 \mathrm{~mm} \times 4.6 \mathrm{~mm}, 5 \mu \mathrm{m})$, (B) Zorbax Eclipse SB-

C18 $(250 \mathrm{~mm} \times 4.6 \mathrm{~mm}, 5 \mu \mathrm{m})$ and (C) Zorbax Eclipse SB-C18 $(100 \mathrm{~mm}$ $\times 2.1 \mathrm{~mm}, 1.8 \mu \mathrm{m})$

Additional file 9: Regression equation using different columns

Columns: Zorbax Eclipse SB-C18 $(250 \mathrm{~mm} \times 4.6 \mathrm{~mm}, 5 \mu \mathrm{m})$ and Zorbax Eclipse SB-C18 $(100 \mathrm{~mm} \times 2.1 \mathrm{~mm}, 1.8 \mu \mathrm{m})$

\section{Abbreviations}

XST: Xuesaitong; HPLC-UV: high performance liquid chromatographyultraviolet detection; HPLC-PDA/ESI-MS ${ }^{n}$ : HPLC with photo diode array detection/electrospray ionization tandem mass spectrometry; HPLC-ELSD: high performance liquid chromatography-evaporative light scattering detection; HPLC-MS: high performance liquid chromatography-mass spectroscopy; SFDA: State Food and Drug Administration (China)

\section{Acknowledgements}

This work was supported by the National S\&T Major Project (No. 2009ZX09502-005 \& 2009ZX09311-002) and Zhejiang Provincial Natural Science Foundation, China (R2080693).

\section{Authors' contributions}

XHF designed the study. HY performed the fingerprint and quantitative analysis and wrote the manuscript. PYS and QS assisted HY to identify the characteristic peaks using HPLC-PDA/ESI-MS ${ }^{n}$. All authors read and approved the final version of the manuscript.

\section{Competing interests}

The authors declare that they have no competing interests.

Received: 29 July 2010 Accepted: 24 February 2011 Published: 24 February 2011

\section{References}

1. Lau AJ, Woo SO, Koh HL: Analysis of saponins in raw and steamed Panax notoginseng using high-performance liquid chromatography with diode array detection. J Chromatogr A 2003, 1011:77-87.

2. Lau AJ, Seo BH, Woo SO, Koh HL: High-performance liquid chromatographic method with quantitative comparisons of whole chromatograms of raw and steamed Panax notoginseng. J Chromatogr A 2004, 1057:141-149. 
3. Li L, Zhang JL, Sheng YX, Guo DA, Wang Q, Guo HZ: Simultaneous quantification of six major active saponins of Panax notoginseng by high-performance liquid chromatography-UV method. I Pharm Biomed Anal 2005, 38:45-51.

4. Guan J, Lai CM, Li SP: A rapid method for the simultaneous determination of 11 saponins in Panax notoginseng using ultra performance liquid chromatography. J Pharm Biomed Anal 2007, 44:996-1000

5. Qian ZM, Wan JB, Zhang QW, Li SP: Simultaneous determination of nucleobases, nucleosides and saponins in Panax notoginseng using multiple columns high performance liquid chromatography. J Pharm Biomed Anal 2008, 48:1361-1367.

6. Wan JB, Yang FQ, Li SP, Wang YT, Cui XM: Chemical characteristics for different parts of Panax notoginseng using pressurized liquid extraction and HPLC-ELSD. J Pharm Biomed Anal 2006, 41:1596-1601.

7. Wang XY, Zhao T, Gao XF, Dan M, Zhou MM, Jia W: Simultaneous determination of 17 ginsenosides in rat urine by ultra performance liquid chromatography-mass spectrometry with solid-phase extraction. Anal Chim Acta 2007, 594:265-273.

8. Lai CM, Li SP, Yu H, Wan JB, Kan KW, Wang YT: A rapid HPLC-ESI-MS/MS for qualitative and quantitative analysis of saponins in "XUESETONG" injection. J Pharm Biomed Anal 2006, 40:669-678.

9. Li L, Tsao R, Dou JP, Song FR, Liu ZQ, Liu SY: Detection of saponins in extract of Panax notoginseng by liquid chromatography-electrospray ionisation-mass spectrometry. Anal Chim Acta 2005, 536:21-28.

10. Li XY, Sun JG, Wang GJ, Hao HP, Liang Y, Zheng YT, Yan B, Sheng LS: Simultaneous determination of panax notoginsenoside $R_{1}$, ginsenoside $\mathrm{Rg}_{1}, \mathrm{Rd}, \mathrm{Re}$ and $\mathrm{Rb}_{1}$ in rat plasma by HPLC/ESI/MS: platform for the pharmacokinetic evaluation of total panax notoginsenoside, a typical kind of multiple constituent traditional Chinese medicine. Biomed Chromatogr 2007, 21:735-746.

11. Liu HL, Xia L, Cao J, Li P, Qi LW: Simultaneous determination of twelve saponins in Radix et Rhizoma Notoginseng by rapid resolution LC-ESITOF-MS. Chromatographia 2008, 68:1033-1038.

12. Chan ECY, Yap SL, Lau AJ, Leow PC, Toh DF, Koh HL: Ultra-performance liquid chromatography/time-of-flight mass spectrometry based metabolomics of raw and steamed Panax notoginseng. Rapid Commun Mass Spectrom 2007, 21:519-528.

13. Dan M, Su MM, Gao XF, Zhao T, Zhao AH, Xie GX, Qiu YP, Zhou MM, Liu Z, Jia W: Metabolite profiling of Panax notoginseng using UPLC-ESI-MS. Phytochemistry 2008, 69:2237-2244.

14. Wang XJ, Lv HT, Sun H, Jiang XG, Wu ZM, Sun WJ, Wang P, Liu L, Bi KS: Quality evaluation of Yin Chen Hao Tang extract based on fingerprint chromatogram and simultaneous determination of five bioactive constituents. J Sep Sci 2008, 31:9-15.

15. Liu AH, Lin YH, Yang M, Guo H, Guan SH, Sun JH, Guo DA: Development of the fingerprints for the quality of the roots of Salvia miltiorrhiza and its related preparations by HPLC-DAD and LC-MS ${ }^{n}$. J Chromatogr B 2007, 846:32-41.

16. Han C, Shen Y, Chen JH, Lee FSC, Wang XR: HPLC fingerprinting and LCTOF-MS analysis of the extract of Pseudostellaria heterophylla (Miq.) Pax root. J Chromatogr B 2008, 862:125-131.

17. Qiao CF, Han QB, Song JZ, Mo SF, Kong LD, Kung HF, Xu HX: Chemical fingerprint and quantitative analysis of Fructus Psoraleae by highperformance liquid chromatography. J Sep Sci 2007, 30:813-818.

18. Ding S, Dudley E, Plummer S, Tang J, Newton RP, Brenton AG: Fingerprint profile of Ginkgo biloba nutritional supplements by LC/ESI-MS/MS. Phytochemistry 2008, 69:1555-1564.

19. Jiang Y, Li SP, Wang YT, Chen XJ, Tu PF: Differentiation of Herba Cistanches by fingerprint with high-performance liquid chromatography-diode array detection-mass spectrometry. J Chromatogr A 2009, 1216:2156-2162.

20. Jin XF, Lu YH, Wei DZ, Wang ZT: Chemical fingerprint and quantitative analysis of Salvia plebeia R.Br. by high-performance liquid chromatography. J Pharm Biomed Anal 2008, 48:100-104.

21. Kong WJ, Zhao YL, Xiao XH, Jin C, Li ZL: Quantitative and chemical fingerprint analysis for quality control of Rhizoma Coptidischinensis based on UPLC-PAD combined with chemometrics methods. Phytomedicine 2009, 16:950-959.

22. Li W, Deng YL, Dai RJ, Yu YH, Saeed MK, Li L, Meng WW, Zhang XS: Chromatographic fingerprint analysis of Cephalotaxus sinensis from various sources by high-performance liquid chromatography-diodearray detection-electrospray ionization-tandem mass spectrometry. J Pharm Biomed Anal 2007, 45:38-46.

23. Dumarey $M$, van Nederkassel AM, Deconinck E, Vander Heyden Y: Exploration of linear multivariate calibration techniques to predict the total antioxidant capacity of green tea from chromatographic fingerprints. J Chromatogr A 2008, 1192:81-88.

24. Teo CC, Tan SN, Yong JWH, Hew CS, Ong ES: Validation of green-solvent extraction combined with chromatographic chemical fingerprint to evaluate quality of Stevia rebaudiana Bertoni. J Sep Sci 2009, 32:613-622.

25. Ni YN, Lai YH, Brandes S, Kokot S: Multi-wavelength HPLC fingerprints from complex substances: An exploratory chemometrics study of the Cassia seed example. Anal Chim Acta 2009, 647:149-158.

26. Li J, Li WZM, Huang W, Cheung AWH, Bi CWC, Duan R, Guo AJY, Dong TTX, Tsim KWK: Quality evaluation of Rhizoma Belamcandae (Belamcanda chinensis (L.) DC.) by using high-performance liquid chromatography coupled with diode array detector and mass spectrometry. J Chromatogr A 2009, 1216:2071-2078.

27. World Health Organization: Guidelines for the Assessment of Herbal Medicines WHO, Munich, Geneva; 1991.

28. State Food and Drug Administration of China: Technical Requirements for the Development of Fingerprints of TCM Injections SFDA, Beijing; 2000.

doi:10.1186/1749-8546-6-9

Cite this article as: Yao et al.: Chemical fingerprinting and quantitative analysis of a Panax notoginseng preparation using HPLC-UV and HPLCMS. Chinese Medicine 2011 6:9.

\section{Submit your next manuscript to BioMed Central and take full advantage of:}

- Convenient online submission

- Thorough peer review

- No space constraints or color figure charges

- Immediate publication on acceptance

- Inclusion in PubMed, CAS, Scopus and Google Scholar

- Research which is freely available for redistribution

Submit your manuscript at www.biomedcentral.com/submit
Biomed Central 